\title{
Evaluation of Prostatic Lesions by Transrectal Ultrasonography, Colour Doppler and TRUS-Guided Biopsy
}

\author{
Anubha Singh ${ }^{1}$, Kritika Agrawal2 ${ }^{2}$, Sandeep Tyagi ${ }^{3}$, Fawaz Yousuf4 ${ }^{4}$, Astha Garg ${ }^{5}$ \\ 1, 2, 3, 4, 5 Department of Radiodiagnosis, Maharishi Markandeshwar Institute of \\ Medical Sciences and Research (MMIMSR), Mullana, Haryana, India.
}

\section{ABSTRACT}

\section{BACKGROUND}

We wanted to evaluate the role of a transrectal ultrasound (TRUS) guided biopsy and a colour Doppler test in the detection of prostate cancer in patients with increased serum prostate-specific antigen (PSA) levels and / or an abnormal digital rectal examination (DRE).

\section{METHODS}

A total of 30 cases, in the age group of 50 to 80 years and showing increased serum PSA levels ( $>4 \mathrm{ng} / \mathrm{mL}$ ) and / or abnormal DRE, was enrolled. They underwent TRUS evaluation followed by colour Doppler flowmetric studies. A TRUS-guided fine needle aspiration cytology (FNAC) / biopsy was performed in all the cases. The findings were confirmed histopathologically. Data was analysed using the chi-square and Fisher exact test.

\section{RESULTS}

Histopathologically, a total of 11 cases (36.66 \%) were malignant. On TRUS, irregular shape, heterogeneous echotexture loss of differentiation between the peripheral and internal zones, and capsular invasion were significantly associated with malignancy. On flowmetry, moderate vascularity and focal asymmetry were significantly associated with malignancy. The combined use of TRUS and colour Doppler flowmetry was found to be $100 \%$ sensitive and $89.5 \%$ specific and had a positive predictive value (PPV) and a negative predictive value (NPV) of $84.6 \%$ and $100 \%$, respectively.

\section{CONCLUSIONS}

TRUS with colour Doppler ultrasound plays an important role in the detection of prostatic malignancy with high sensitivity as well as specificity. The high negative predictive value, as observed in the present study could avoid unnecessary diagnostic invasive intervention.

\section{KEY WORDS}

PSA (Prostate Specific Antigen), DRE (Digital Rectal Examination), TRUS (Transrectal Ultrasonography), CAP (Carcinoma Prostate)
Corresponding Author: Dr. Sandeep Tyagi, $3^{\text {rd }}$ G 61, Nehru Nagar, Rakesh Marg Ghaziabad, Uttar Pradesh, India.

E-mail: sandytyagi07@gmail.com

DOI: $10.14260 / j e m d s / 2021 / 14$

How to Cite This Article:

Singh A, Agrawal K, Tyagi $S$, et al. Evaluation of prostatic lesions by transrectal ultrasonography, colour doppler and trus guided biopsy. J Evolution Med Dent Sci 2021;10(02):68-73, DOI: 10.14260/jemds/2021/14

Submission 18-09-2020,

Peer Review 14-11-2020,

Acceptance 20-11-2020,

Published 11-01-2021.

Copyright @ 2021 Anubha Singh et al. This is an open access article distributed under Creative Commons Attribution License [Attribution 4.0 International (CC BY 4.0)] 


\section{BACKGROUND}

Prostate cancer is the most common malignant tumour in men all over the world. Incidence of prostatic cancer increases proportionally after the age of 50 years. ${ }^{1}$ In approximately, 70 $\%$ of cases, it arises in the peripheral zone of gland particularly in the posterior location. ${ }^{2}$ Adenocarcinoma is its most common histological variant. ${ }^{1}$

Carcinoma Prostate (CAP) is globally the second most frequently diagnosed cancer and the sixth leading cause of cancer deaths in males. The worldwide incidence of CAP has been rising rapidly, likely due to intensified effort in early detection and screening. ${ }^{3}$

$95 \%$ of prostate cancers are adenocarcinomas which develop from the acini of the prostatic ducts. Acinar-type adenocarcinoma is the most common malignancy of the prostate, comprising more than $90 \%$ of malignant lesions. ${ }^{4}$ Acinar type adenocarcinoma typically involves the peripheral zones and is associated with high-grade prostatic intraepithelial neoplasia (HGPIN) which is the only recognized premalignant prostate lesion. Prostate Specific Antigen (PSA) is currently the only serological marker routinely used in the diagnosis, staging and monitoring of treatment response or failure in prostate cancer. ${ }^{5}$ PSA remains a better predictor of prostate cancer than Digital Rectal Examination (DRE) or transrectal ultrasound. TRUS with or without biopsy and to a lesser extent DRE is also good predictor for diagnosing CAP. Prostate biopsy remains the gold standard investigation for diagnosing prostate cancer. ${ }^{5}$

Prostate cancer often develops very slowly compared with most other cancers and the natural history often spans several decades. However, some cancers grow fast and will give rise to early metastases and death. As far as diagnosis is concerned, it is still difficult to differentiate those prostate cancers that remain indolent and those that will proliferate more quickly. There is no fixed pattern of proliferation; it is often multifocal and usually grows locally. Prostate cancer can infiltrate and penetrate the prostatic (pseudo) capsule; often penetration is basal and lateral and continues towards the seminal vesicles or follows the neurovascular bundles. ${ }^{6}$ It may spread into regional lymph nodes to finally give rise to distant metastases. The risk of lymph node metastasis increases with increased tumour volume and poor histological differentiation.

Prostate cancer can spread by local invasion (typically into the bladder and seminal vesicles, urethral and rectal involvement are rare), lymphatic spread (pelvic nodes first followed by para-aortic and inguinal nodes), or by haematogenous metastases.

PSA is a tissue and not tumour-specific marker, which is important because elevated levels of PSA may occur in instrumentation of the urinary tract, benign prostatic enlargement (BPE), urinary tract infection, prostatitis, acute urinary retention or large urinary residual. ${ }^{5}$

Transrectal ultrasonography (TRUS) has received increasing attention recently because of its potential for early detection of prostate cancer. It provides greater detail of zonal anatomy of prostate and echo pattern of the gland and its various lesions. The prostate gland can be visualized with a transrectal probe allowing close-contact scanning. Ultrasound is essential for examining the echotexture and size of the gland and to aid precision biopsies. It is more accurate than DRE examination in measuring prostate size. It is routine to measure the prostate volume which may be important in offering treatment options. The prostate is measured in 3 planes. In the transverse view 1) anterior to posterior (width) 2) height and in the longitudinal plane 3) from the bladder neck to the apex (length). In evaluation for suspicion of prostate cancer, TRUS also provides information regarding the existence and location of cancer foci. Prostate cancer is typically visualized as a hypoechoic lesion in the peripheral zone, and at times it is accompanied by asymmetry or protrusion into the prostatic capsule. In advanced disease, asymmetry between the seminal vesicles is seen through TRUS. The limitation of TRUS is the reliability of the findings is operator dependent, and TRUS suffers from poor test characteristics for the diagnosis of prostate cancer.

Transrectal ultrasound (TRUS) is generally recognized as the method of choice for prostatic biopsy guidance, however, only $20 \%$ of urologists perform targeted biopsies based on sonographic findings. Most contemporary prostate biopsy protocols have concentrated on the use of the systematic prostate biopsy approach and currently require the acquisition of 10 or more cores. This investigation though sensitive and specific, however, is very painful and invasive procedure rendering it unfit for screening. ${ }^{7}$ Moreover, the low positive predictive value (PPV) for the presence of prostate cancer remains a considerable weakness. Doppler and grayscale ultrasound are the two modes generally used to examine the prostate after contrast administration. Echogenic material within the vasculature is visible on gray-scale and the Doppler signal is greatly enhanced, particularly within areas of cancer. Colour and power Doppler biopsy targeted at such areas of abnormal flow are more sensitive than random biopsy. Colour Doppler ultrasonography is an adjunct technique to traditional gray-scale imaging. It is designed to help clinicians better identify areas of increased vascularity that is associated with tumours. Malignant lesions are characterized by asymmetrically increased flow patterns, particularly in highergrade prostate cancers with a Gleason score of above 8. Colour Doppler ultrasonography (CDUS) correlates positively with the grade and stage, as well as biochemical recurrence of prostate cancer, after initial treatment.

Although increased cancer detection has been reported for the use of colour Doppler US, the combined sensitivity of grayscale and colour Doppler imaging is insufficient to preclude the need for systemic biopsies. ${ }^{8}$

Hence, there is a need for an alternate, cost effective and efficient modality of screening, detection and differentiation of prostatic diseases; the present study is done to study the role of "transrectal ultrasonography (TRUS) and colour doppler to evaluate the prostatic lesions and comparing this modality with the histopathology".

\section{METHODS}

The study was a prospective observational study, conducted in the Department of Radiodiagnosis, Maharishi Markandeshwar Institute of Medical Sciences and Research (MMIMSR), Mullana from November 2017 to June 2019. Total number of patients referred from surgery department, fulfilling our inclusion criteria during the period of our study were 36 out of whom 30 were willing for the TRUS guided biopsy procedure needed for the histopathological correlation of our study. 
Hence the study was carried out on a total of 30 patients. The study was conducted after approval from institutional ethics committee, MMIMSR, Mullana. All suspected patients attending surgical outpatient / inpatients department who fulfilled the inclusion criteria of age $>50$ years, raised PSA levels and having a hard and enlarged nodular prostate on DRE were thoroughly examined by general physical examination and systemic examination followed by TRUS with a colour Doppler for the detection of prostatic lesion. TRUS was done on Philips HD-15 ultrasonography (USG) colour Doppler machine (with a transrectal probe (6-10 MHz)), in the proper position (knee chest position). To assure acoustic contact the sheath contained ultrasound gel. The sheath coated with gel for adequate lubrication was inserted into the rectum. Then FNAC / biopsy was taken under TRUS guidance from the suspected lesions.

On TRUS, the number of nodules, zone involved, size and shape of the lesion, echogenicity, and difference between peripheral and internal zone, prostate weight, and capsular invasion were noted. Doppler colour flowmetry studies were done for the extent of vascularity (mild / moderate) and vascular asymmetry (focal / diffuse). Then FNAC / biopsy was taken under TRUS guidance from the suspected lesions.

\section{Statistical Analysis}

Collected data was analysed for sensitivity, specificity, positive predictive value, negative predictive value and accuracy by applying the $\mathrm{p}$ value in chi square and Fisher exact test to assess the statistical significance.

\section{RESULTS}

The age of patients in present study ranged from 51 years to 80 years $(n=30)$. The mean age was 67.53 years. Most of the patients were in the age group of $71-80$ years $(n=14)$. In our study, with increase in age, there is increase in frequency of prostatic disease. The main clinical presentation of the patients was nocturia (26.7\%) and acute retention (26.7\%) followed by frequency and hesitancy (23.3\%).

Of the 30 patients, 12 patients ( $40 \%$ ) had nodularity on digital rectal examination and 10 patients (33.3\%) had induration. 6 patients had PSA value $(4-5 \mathrm{ng} / \mathrm{mL}), 10$ patients had PSA level $(15-50 \mathrm{ng} / \mathrm{mL})$ and 14 had PSA value $>50 \mathrm{ng} / \mathrm{mL}$, minimum serum PSA level of the patients was $5.14 \mathrm{ng} / \mathrm{mL}$ whereas the maximum serum PSA level was $168.73 \mathrm{ng} / \mathrm{mL}$. The mean serum PSA level of the patients was $54.59 \mathrm{ng} / \mathrm{mL}$. Raised PSA levels were found in concordance with malignancy. 12 (40 \%) out of 30 patients had heterogeneous echotexture of prostatic tissue on TRUS, of whom 10 patients were malignant on histology and 2 patients were benign whereas $18(60 \%)$ out of 30 patients had homogenous echotexture, of whom only 1 patient was malignant on histology.

In our study of 30 patients, on TRUS examination we found that the differentiation between peripheral and internal zone were lost in 10 patients (33.3\%). Of whom, 8 patients $(80 \%)$ had malignant lesion proven on histology. Out of 20 patients $(66.7 \%)$ with maintained differentiation between peripheral and internal zone $2(10 \%)$ were found to be malignant. Hence, proving the significance of the study that loss of differentiation between peripheral and internal zone is associated with malignancy ( $\mathrm{p}$ value $<0.01$ ).

On TRUS examination, out of 30 patients 6 patients (20\%) showed capsular invasion and peripheral spread, and they were positive for malignant histology.

TRUS done for zonal distribution in 24 patients with focal lesions (out of 30 patients 24 had focal lesions) showed that out of 3 patients (12.5\%) with focal lesion found at apex, none were found to be malignant. Out of 7 patients $(27.2 \%)$ with focal lesion found at basal region, 1 (14.3\%) was found malignant. Out of 12 patients (50\%) with lesion found in peripheral zone unilaterally, $4(33.3 \%)$ were found to be malignant. Out of 2 patients $(8.3 \%)$ with lesion found in transitional zone unilaterally, none were found to be malignant. The location of the lesion in peripheral zone was significantly associated with the malignancy of prostate $(\mathrm{p}<$ 0.01).

Considering the sizes of focal lesion, out of 15 patients $(62.5 \%)$ showing lesions of size $<2 \mathrm{~cm} 3(20 \%)$ were found to be malignant, out of 8 patients $(33.5 \%)$ showing lesions of sizes $2-5 \mathrm{~cm}$, one $(12.5 \%)$ was found to be malignant, the single patient showing lesion of size $>5 \mathrm{~cm}$ was found to be malignant (100\%).

Considering the shape of the nodule, out of 6 patients $(25$ $\%)$ showing irregular nodules $4(66.7 \%)$ were found to be malignant, out of 12 patients (50\%) showing regular and round shaped nodules none were found to be malignant and out of 6 patients ( $25 \%$ ) showing oval shaped nodules only one was found to be malignant (16.7\%). Therefore, the nodules appearing irregular in shape were more consistently associated with malignancy $(\mathrm{p}<0.03)$.

On the basis of echo pattern of the focal lesions, out of 11 patients $(45.8 \%)$ showing hypoechoic nodules on TRUS 5 $(45.5 \%)$ were found to be malignant, out of the remaining 13 patients $(54.2 \%)$, none of the lesions were found to be malignant. Hence, the study was found to be significant as all the malignant lesions appeared hypoechoic on TRUS ( $p<$ 0.01).

Out of 30 patients, 24 were found to have single or multiple focal lesions on TRUS evaluation. Of whom 5 (20.8\%) were found to be malignant and remaining 6 patients (20\%) showing no focal lesion but appearing heterogeneous in echotexture and showing loss of differentiation between peripheral and transitional zone and capsular invasion, all were found to be malignant. Hence, proving that the features such as heterogeneous echotexture, loss of differentiation between peripheral and transitional zone and capsular invasion are more associated with malignancy as compared to a focal lesion itself ( $\mathrm{p}$ value $<0.01$ ).

On colour Doppler assessment, 28 patients showed abnormal vascularity. 15 patients (50 \%) showed abnormal moderate vascularity out of whom $90.9 \%$ patient have malignant lesion. 13 patients (43.3\%) showed abnormal focal vascularity out whom $90 \%$ patient have malignant lesion suggesting vascularity to be significantly associated with malignancy.

Overall, out of the 30 patients referred for TRUS 17 patients $(56.7 \%)$ were diagnosed as benign prostatic hyperplasia and 13 patients (43.3\%) were diagnosed to have malignancy on TRUS evaluation. After histopathological examination 19 cases (63.3\%) were found to be benign prostatic hyperplasia, 11 cases (36.7 \%) were malignant (out of which 2 cases $(6.7 \%)$ were carcinoma in situ). 
On clinical examination, abnormal DRE, and PSA level $>4$ ng / mL, 19 patients were suspected of having malignant lesions, after histopathological examination we concluded that clinical diagnosis had a diagnostic accuracy of $73.3 \%$ in determining prostatic malignancy with a specificity of $57.9 \%$, sensitivity of $100 \%$, PPV of $57.9 \%$ and NPV of $100 \%$.

Hence, concluding that the combination of TRUS and colour Doppler has resulted in increased specificity and accuracy as compared to clinical diagnosis. The sensitivity of the combined evaluation appears to be $100 \%$ and the specificity has been shown to lie between $66.9 \%$ - $98.7 \%$. Colour Doppler improves the sensitivity of endorectal ultrasound in spite of some false negative results. It demonstrates tumour vascularity, detect capsular extension and aids in image guided FNAC / biopsy. TRUS with colour Doppler had diagnostic accuracy of $93.3 \%$, PPV of $84.6 \%$ and NPV of $100 \%$

\begin{tabular}{|c|c|c|}
\hline Sl. No. & Characteristics & Statistics \\
\hline 1 & $\begin{array}{c}\text { Mean age } \pm \text { SD (range) in years } \\
\text { Most of the patients were in age group } 71-80 \\
\text { years }(n=14)\end{array}$ & $67.53 \pm 8.36$ \\
\hline 2 & DRE findings & \\
\hline \multirow{2}{*}{3} & Induration & $10(33.3 \%)$ \\
\hline & Nodularity & $12(40 \%)$ \\
\hline \multirow[t]{2}{*}{4} & Mean PSA (ng / mL) 54.59 & \\
\hline & $4-15$ & $6(20.0 \%)$ \\
\hline \multirow[t]{2}{*}{5} & $15-50$ & $10(33.3 \%)$ \\
\hline & $>50$ & $14(46.7 .0 \%)$ \\
\hline \multirow[t]{2}{*}{6} & Histopathological diagnosis & \\
\hline & Benign & $19(63.3 \%)$ \\
\hline \multirow[t]{2}{*}{7} & Carcinoma prostate & $9(30 \%)$ \\
\hline & Carcinoma in situ & $2(6.7 \%)$ \\
\hline 8 & TRUS (grey scale diagnosis) & \\
\hline \multirow{2}{*}{9} & Benign & $17(56.7 \%)$ \\
\hline & Malignant & $13(43.3 \%)$ \\
\hline 10 & TRUS + colour Doppler & \\
\hline 11 & Normal vascularity & $2(6.7 \%)$ \\
\hline 12 & Moderate vascularity & $15(50 \%)$ \\
\hline 13 & Focal vascularity & $13(43.3 \%)$ \\
\hline \multicolumn{3}{|c|}{ Table 1. General and Clinical Profiles of Patients } \\
\hline
\end{tabular}

\begin{tabular}{|c|c|c|}
\hline $\begin{array}{l}\text { Sl. } \\
\text { No. }\end{array}$ & Characteristics & Statistics \\
\hline 1 & $\begin{array}{c}\text { Mean age } \pm \text { SD (range) in years } \\
\text { Most of the patients were in age group } 71-80 \\
\text { years }(n=14)\end{array}$ & $67.53 \pm 8.36$ \\
\hline 2 & DRE findings & \\
\hline \multirow{6}{*}{3} & Induration & $10(33.3 \%)$ \\
\hline & Nodularity & $12(40 \%)$ \\
\hline & Mean PSA (ng / mL) 54.59 & \\
\hline & $4-15$ & $6(20.0 \%)$ \\
\hline & $15-50$ & $10(33.3 \%)$ \\
\hline & $>50$ & $14(46.7 .0 \%)$ \\
\hline \multirow[t]{4}{*}{4} & Histopathological diagnosis & \\
\hline & Benign & $19(63.3 \%)$ \\
\hline & Carcinoma prostate & $9(30 \%)$ \\
\hline & Carcinoma in situ & $2(6.7 \%)$ \\
\hline \multirow[t]{3}{*}{5} & TRUS (grey scale diagnosis) & \\
\hline & Benign & $17(56.7 \%)$ \\
\hline & Malignant & $13(43.3 \%)$ \\
\hline \multirow[t]{4}{*}{6} & TRUS + colour doppler & \\
\hline & Normal vascularity & $2(6.7 \%)$ \\
\hline & Moderate vascularity & $15(50 \%)$ \\
\hline & Focal vascularity & $13(43.3 \%)$ \\
\hline \multicolumn{3}{|c|}{$\begin{array}{l}\text { Table 2. TRUS Finding for Malignant Lesion } \\
\text { on Radiological Findings }(n=13)\end{array}$} \\
\hline
\end{tabular}

Focal lesion with irregular shape, hypoechoic nodules with moderate and focal vascularity were considered malignant. In cases where focal lesions were not present, heterogeneous echotexture with loss of differentiation between peripheral and transitional zone and capsular invasion were considered as malignant features.

\begin{tabular}{|c|c|c|c|c|c|c|c|}
\hline \multirow{2}{*}{ Characteristics } & \multicolumn{2}{|c|}{ Total } & \multicolumn{2}{|c|}{ Benign } & \multicolumn{2}{|c|}{ Malignant } & \multirow{2}{*}{$\begin{array}{c}\mathbf{P} \\
\text { Value }\end{array}$} \\
\hline & No. & $\%$ & No. & $\%$ & No. & $\%$ & \\
\hline \multicolumn{7}{|c|}{ No. of nodules } & \multirow{4}{*}{0.02} \\
\hline 1 & 11 & 45.8 & 9 & 81.8 & 2 & 18.2 & \\
\hline 2 & 05 & 20.8 & 3 & 60 & 2 & 40 & \\
\hline$>2$ & 08 & 33.3 & 7 & 87.5 & 1 & 12.5 & \\
\hline \multicolumn{7}{|c|}{ Zone } & \multirow{5}{*}{0.01} \\
\hline Apex & 3 & 12.5 & 3 & 100 & 0 & 0.0 & \\
\hline Basal & 7 & 29.2 & 6 & 85.7 & 1 & 14.3 & \\
\hline Unilateral peripheral & 12 & 50.0 & 8 & 66.7 & 4 & 33.3 & \\
\hline Unilateral transitional & 2 & 8.3 & 2 & 100 & 0 & 0.0 & \\
\hline \multicolumn{8}{|c|}{ Size } \\
\hline$<2 \mathrm{~cm}$ & 15 & 62.5 & 12 & 80 & 3 & 20 & \multirow{3}{*}{0.06} \\
\hline $2-5 \mathrm{~cm}$ & 08 & 33.3 & 7 & 87.5 & 1 & 12.5 & \\
\hline$>5 \mathrm{~cm}$ & 01 & 4.2 & 0 & 0.0 & 1 & 100 & \\
\hline \multicolumn{8}{|c|}{ Shape } \\
\hline Irregular & 06 & 25 & 2 & 33.3 & 4 & 66.7 & \multirow{4}{*}{0.03} \\
\hline Regular & 06 & 25 & 6 & 100 & 0 & 0.0 & \\
\hline Round & 06 & 25 & 6 & 100 & 0 & 0.0 & \\
\hline Oval & 06 & 25 & 5 & 83.3 & 1 & 16.7 & \\
\hline \multicolumn{8}{|c|}{ Echo pattern of Focal Lesion } \\
\hline Hypoechoic & 11 & 45.8 & 6 & 54.5 & 05 & 45.5 & \multirow[b]{3}{*}{$<0.01$} \\
\hline Isoechoic & 01 & 4.2 & 1 & 100 & 0 & 0.0 & \\
\hline Iso to hypoechoic & 12 & 50 & 12 & 100 & 0 & 0.0 & \\
\hline \multicolumn{8}{|c|}{ Table 3. Characteristics of Focal Lesions on TRUS $(n=24)$} \\
\hline & \multicolumn{5}{|c|}{ Histopathological Diagnosis } & \multirow{2}{*}{\multicolumn{2}{|c|}{ Total }} \\
\hline TRUS Diagnosis & \multirow{2}{*}{\multicolumn{2}{|c|}{$\begin{array}{l}\text { Benign } \\
17(\mathrm{TN})\end{array}$}} & \multicolumn{3}{|c|}{ Malignant } & & \\
\hline Benign & & & \multicolumn{3}{|c|}{$0(\mathrm{FN})$} & \multicolumn{2}{|c|}{17} \\
\hline Malignant & \multicolumn{2}{|c|}{$02(\mathrm{FP})$} & & 11 (TP) & & \multicolumn{2}{|c|}{13} \\
\hline Total & \multicolumn{2}{|c|}{19} & & 11 & & \multicolumn{2}{|c|}{30} \\
\hline
\end{tabular}

- $\quad$ Sensitivity $=100.0 \%(71.5 \%-100 \%)$

- $\quad$ Specificity $=17 / 19=89.5 \%(66.9 \%-98.7 \%)$

- $\quad \mathrm{PPV}=84.6 \%(59.7 \%-95.3 \%)$

- $\quad \mathrm{NPV}=100.0 \%$

- $\quad$ Accuracy $=93.3 \%(77.9 \%-99.2 \%)$

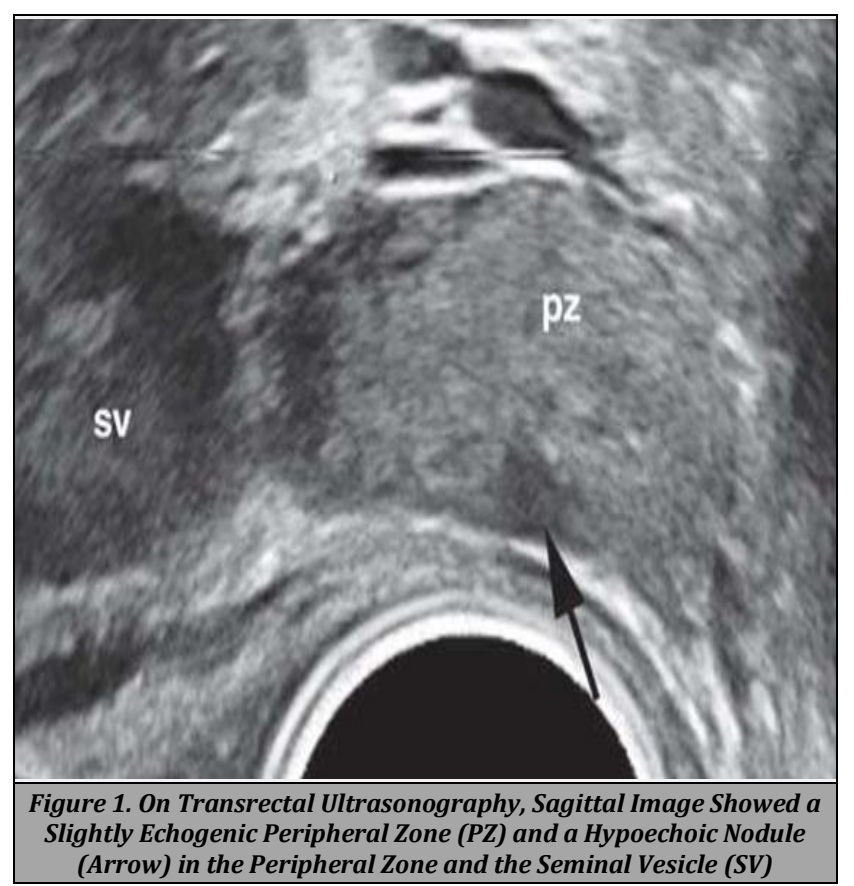




\section{DISCUSSION}

The diagnosis and treatment of prostate cancer are very challenging. The current methods of screening for prostate cancer include measuring serum prostate specific antigen levels (PSA), digital rectal examination (DRE) and transrectal ultrasound (TRUS). Scanning and biopsy confirms the diagnosis; however, the sensitivity and specificity of TRUS for diagnosing prostate cancer still needs further study.

The mean age of patients in present study was 67.53 years, range $71-80$ years $(n=14)$. In studies conducted by Vilanova et al. (2011), ${ }^{9}$ Aydin et al. (2012) ${ }^{10}$, Sakarya et al. (1998) ${ }^{11}$ and Khanduri et al.,12 the study population consisted men with mean age 63.5 years (range 43 - 87 years), 69 years, 66.4 years range (59- 82 years) and 63.8 years (range 51 to 77 years) respectively. Thus, we can conclude by our study and other studies that with increase in age, there is increase in frequency of prostatic disease. Raised PSA levels were in concordance with malignancy which is similar to study done by Prcic et al. ${ }^{13}$ in which cancer was diagnosed in $57.1 \%$ who had PSA levels more than $>20 \mathrm{ng} / \mathrm{mL}$.

$40 \%$ patients had heterogeneous echotexture of prostatic tissue on TRUS, of which $83.3 \%$ were malignant on histology. Thus, concluding that heterogenous echotexture of prostate had significant association with malignancy which was similar to the study done by Khanduri et al.

In this study, lesions which were present in peripheral zone, size more than $5 \mathrm{~cm}$, irregular in shape and hypoechoic in echotexture were more in the favour of malignancy. In a study done by Ahmed et al.,14 found that majority of patients with hypoechoic nodules, $56.3 \%$ had malignant histology. A study done by Lee et al. ${ }^{15}$ determined that shape, margin and irregularity and vascularity were factors significantly associated with malignancy. In study by Khanduri et al.,12 $61.54 \%$ patients of size $>5 \mathrm{~cm}$ had malignant lesion, $76.92 \%$ patients having irregular shape showed malignant lesion and $100 \%$ of patients with round shape showed benign lesion.

Differentiation between peripheral and internal zone were lost in 10 out of 30 patients (33.3\%) in our study. Out of which 8 patients ( $80 \%$ ) had malignant lesion which was similar to the study done by Khanduri et al. in which $76.92 \%$ patients with malignant lesion showed loss of differentiation. Increased vascularity of the focal lesion was more in the favour of malignancy which was similar to the Lee et al.15 in which $57 \%$ of patients with positive colour Doppler finding were malignant. In our study on TRUS examination, 6 (20\%) out of 30 patients had capsular invasion and peripheral spread of which $100 \%$ showed positive malignant histology which was similar to the study done by Sperandeo et al. ${ }^{16}$ and Ahmed et al. ${ }^{14}$

In our study, we concluded that TRUS with colour Doppler had high sensitivity (100\%), specificity (89.5\%) and high negative predictive value (NPV) (100\%) which was comparable with a study done by Khanduri et asl.

\section{CONCLUSIONS}

TRUS with colour Doppler ultrasound plays an important role in detection of prostatic malignancy with high sensitivity as well as specificity. The high negative predictive value, as observed in the present study, could avoid unnecessary diagnostic invasive intervention. Small sample size is a limitation of this study.

Data sharing statement provided by the authors is available with the full text of this article at jemds.com.

Financial or other competing interests: None.

Disclosure forms provided by the authors are available with the full text of this article at jemds.com.

\section{REFERENCES}

[1] Anunobi CC, Akinde OR, Elesha SO, et al. Prostate diseases in Lagos, Nigeria: a histologic study with tPSA correlation. Niger Postgrad Med J 2011;18(2):98-104.

[2] Hameed S, Malik A, Bilal S, et al. Pattern of prostatic disease; a histopathological survey. Professional Med J 2010;17(4):573-7.

[3] Dabir PD, Ottosen P, Hoyer S, et al. Comparative analysis of three- and two-antibody cocktails to AMACR and basal cell markers for the immunohistochemical diagnosis of prostate carcinoma. Diagn Pathol 2012;7:81.

[4] Chang JM, Lee HJ, Lee SE, et al. Pictorial review: unusual tumours involving the prostate: radiological-pathological findings. Br J Radiol 2008;81(971):907-15.

[5] Kronz JD, Allan CH, Shaikh AA, et al. Predicting cancer following a diagnosis of high-grade prostatic intraepithelial neoplasia on needle biopsy: data on men with more than one follow-up biopsy. Am J Surg Pathol 2001;25(8):1079-85.

[6] Blacklock NJ. Surgical anatomy of the prostate. In: Williams DI, Chisholm GD, eds. Scientific foundations of urology. Chicago: William Heinemann Medical Books Ltd., Year Book Medical Publishers 1976:473-85.

[7] Elabbady AA, Khedr MM. Extended 12-core prostate biopsy increases both the detection of prostate cancer and the accuracy of Gleason score. Eur Urol 2006;49(1):49-53.

[8] Kuligowska E, Barish MA, Fenlon HM, et al. Predictors of prostate carcinoma: accuracy of gray-scale and colour doppler US and serum markers. Radiology 2001;220(3):757-64.

[9] Vilanova JC, Barcelo-Vidal C, Comet J, et al. Usefulness of prebiopsy multifunctional and morphologic MRI combined with free-to-total prostate-specific antigen ratio in the detection of prostate cancer. AJR Am J Roentgenol 2011;196(6):W715-22.

[10] Aydin H, Kizilgoz V, Tatar IG, et al. Detection of prostate cancer with magnetic resonance imaging: optimization of T1-weighted, T2-weighted, dynamic-enhanced T1weighted, diffusion-weighted imaging apparent diffusion coefficient mapping sequences and $\mathrm{mr}$ spectroscopy, correlated with biopsy and histopathological findings. J Comput Assit Tomogr 2012;36(1):30-45.

[11] Sakarya ME, Arslan H, Unal O, et al. The role of power doppler ultrasonography in the diagnosis of prostate cancer: a preliminary study. Br J Urol 1998;82(3):386-8.

[12] Khanduri S, Katyal G, Goyal A, et al. Evaluation of prostatic lesions by transrectal ultrasound, colour doppler and the histopathological correlation. Cureus 2017;9(7):e1422. 
[13] Prcic A, Begic E, Hiros M, et al. Usefulness of total PSA value in prostate diseases diagnosis. Acta Inform Med 2016;24(3):156-61.

[14] Ahmed M, Maitama HY, Bello A, et al. Transrectal ultrasound findings in patients with advanced prostate cancer. Ann Nigerian Med 2010;4(2):59-61.

[15] Lee HY, Lee HJ, Byun SS, et al. Classification of focal prostatic lesions on transrectal ultrasound (TRUS) and the accuracy of TRUS to diagnose prostate cancer. Korean J Radiol 2009;10(3):244-51.

[16] Sperandeo G, Sperandeo M, Morcaldi M, et al. Transrectal ultrasonography for the early diagnosis of adenocarcinoma of the prostate: a new maneuver designed to improve the differentiation of malignant and benign lesions. J Urol 2003;169(2):607-10. 